PREPARED FOR THE U.S. DEPARTMENT OF ENERGY, UNDER CONTRACT DE-AC02-76CH03073

PPPL-3628

PPPL-3628

UC-70

\author{
Determination of 3D Equilibria \\ from Flux Surface Knowledge Only \\ by \\ H.E. Mynick and N. Pomphrey
}

November 2001

M

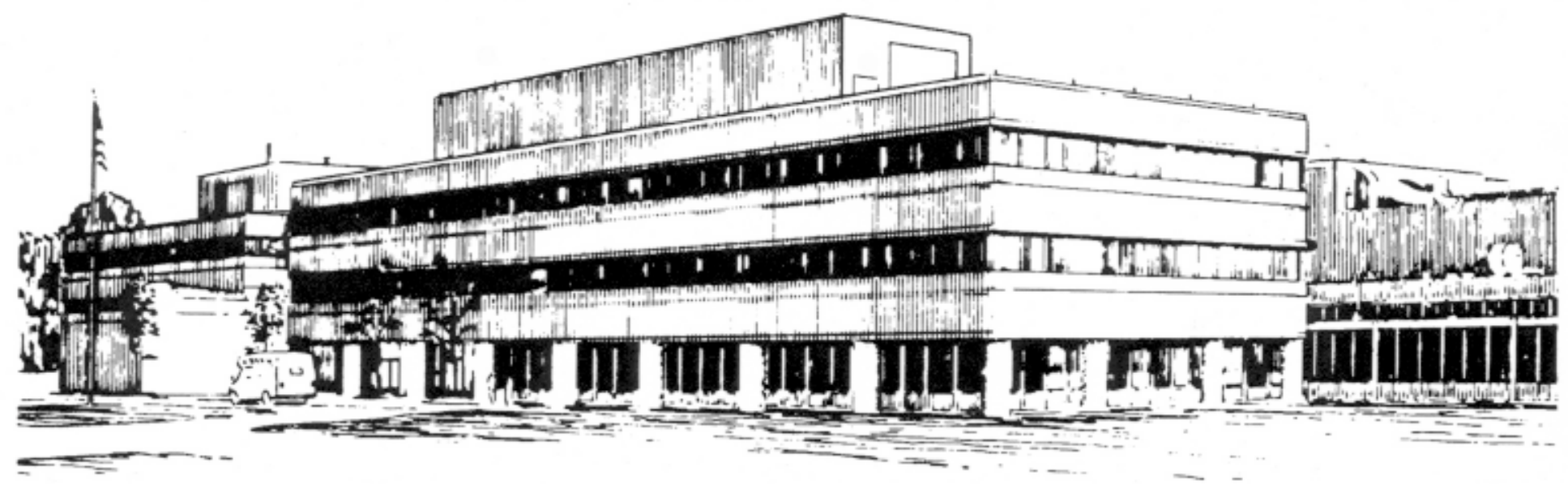

PRINCETON PLASMA PHYSICS LABORATORY PRINCETON UNIVERSITY, PRINCETON, NEW JERSEY 


\section{PPPL Reports Disclaimer}

This report was prepared as an account of work sponsored by an agency of the United States Government. Neither the United States Government nor any agency thereof, nor any of their employees, makes any warranty, express or implied, or assumes any legal liability or responsibility for the accuracy, completeness, or usefulness of any information, apparatus, product, or process disclosed, or represents that its use would not infringe privately owned rights. Reference herein to any specific commercial product, process, or service by trade name, trademark, manufacturer, or otherwise, does not necessarily constitute or imply its endorsement, recommendation, or favoring by the United States Government or any agency thereof. The views and opinions of authors expressed herein do not necessarily state or reflect those of the United States Government or any agency thereof.

\section{Availability}

This report is posted on the U.S. Department of Energy's Princeton Plasma Physics Laboratory Publications and Reports web site in Fiscal Year 2002. The home page for PPPL Reports and Publications is: http://www.pppl.gov/pub_report/

DOE and DOE Contractors can obtain copies of this report from:

U.S. Department of Energy

Office of Scientific and Technical Information

DOE Technical Information Services (DTIS)

P.O. Box 62

Oak Ridge, TN 37831

Telephone: (865) 576-8401

Fax: (865) 576-5728

Email: reports@adonis.osti.gov

This report is available to the general public from:

National Technical Information Service

U.S. Department of Commerce

5285 Port Royal Road

Springfield, VA 22161

Telephone: 1-800-553-6847 or

(703) 605-6000

Fax: (703) 321-8547

Internet: http://www.ntis.gov/ordering.htm 


\title{
Determination of 3D equilibria from flux surface knowledge only
}

\author{
H.E. Mynick, N. Pomphrey \\ Plasma Physics Laboratory, Princeton University \\ P.O. Box 451 \\ Princeton, New Jersey 08543-0451, U.S.A.
}

\begin{abstract}
We show that the method of Christiansen and Taylor, from which complete tokamak equilibria can be determined given only knowledge of the shape of the flux surfaces, can be extended to 3-dimensional equilibria, such as those of stellarators. As for the tokamak case, the given geometric knowledge has a high degree of redundancy, so that the full equilibrium can be obtained using only a small portion of that information.
\end{abstract}

PACS \#s: 52.30.Bt, 52.55.Hc 
Christiansen and Taylor (CT) have shown ${ }^{1}$ that complete MHD equilibria may be obtained for axisymmetric tokamaks with noncircular cross-sections, provided that one initially knows only the shapes of the flux surfaces, described by some flux surface label $\rho(\mathbf{x})$ over real-space $\mathbf{x}$. The demonstration is accomplished by taking appropriate flux-surface averages of the Grad-Shafranov (GS) equation ${ }^{2,3}$ to obtain a simple radial second order o.d.e. for the poloidal flux $\psi$ as a function of $\rho$. This can be solved analytically, and, given $\psi(\rho)$, one can obtain expressions for the pressure gradient profile $p^{\prime} \equiv d p / d \rho$ and poloidal current profile $F$ which appear in the GS equation. Since $\rho(\mathbf{x})$ can be inferred from measurements of physical quantities which are also approximately flux functions (such as density, temperature, or pressure) the method has the potential to be an important diagnostic, and efforts have been made to apply this method to measuring the current and $q$ profiles on JET, ${ }^{4} \mathrm{C}-\mathrm{Mod},{ }^{5}$ and PEGASUS. ${ }^{6}$

It is natural to consider whether such a method also exists for 3-dimensional (3D) toroidal equilibria such as stellarators. In this paper, we demonstrate that this is the case. The GS equation is an elliptic partial differential equation (p.d.e.) in 2 dimensions, usually parametrized by the distance $R$ from the major axis, and the vertical height $Z$ above the midplane, independent of the geometrical toroidal azimuth $\zeta_{g}$ about that axis. Since the derivation of the GS equation makes use of axisymmetry, it is unclear that the method will generalize. However, Degtyarev, et al. ${ }^{7}$ have shown, through insightful choices of flux coordinate systems, that a 3D generalization of the GS equation for $\psi(\mathbf{x})$ exists, which we shall refer to as the 3D-GS equation. Here we show that this more complicated equation may also be subjected to a procedure like that in Ref. 1 to obtain a radial o.d.e. for $\psi(\rho)$ of the same form as in the $2 \mathrm{D}$ case, but with more complicated coefficients. CT have pointed out that their procedure for tokamaks is more robust the more highly 
shaped the tokamak cross section. One might conjecture that, because of the strong poloidal and toroidal shaping of typical stellarators, the CT procedure would in fact be more suited to stellarators than to tokamaks.

We briefly review the origin of the 3D-GS equation. ${ }^{7}$ In a general flux coordinate system $\left\{q^{i}\right\} \equiv\{\rho, \theta, \zeta\}$ (for $i=1,2,3$ ) parametrizing a torus, with poloidal angle $\theta$, toroidal angle $\zeta$, and flux surface label $\rho$ already introduced, one may represent the magnetic field in both the contravariant (Clebsch) representation,

$$
\mathbf{B}=\nabla \psi \times \nabla \zeta+\nabla \Phi \times \nabla \theta=\mathbf{e}_{\theta} B^{\theta}+\mathbf{e}_{\zeta} B^{\zeta},
$$

and in the covariant representation

$$
\mathbf{B}=J \nabla \theta+F \nabla \zeta-\nu \nabla \rho+\nabla \phi .
$$

Here, the $\mathbf{e}_{i} \equiv \mathcal{J} \mathbf{e}^{j} \times \mathbf{e}^{k}$ are the contravariant basis vectors, where $i, j$, and $k$ are cyclic. These are reciprocal to the covariant set $\mathbf{e}^{i}$, usually taken equal to $\nabla q^{i}$, in which case $\mathbf{e}_{i}=\partial \mathbf{x} / \partial q^{i} . \mathcal{J} \equiv\left(\mathbf{e}^{1} \cdot \mathbf{e}^{2} \times \mathbf{e}^{3}\right)^{-1}=\mathbf{e}_{1} \cdot \mathbf{e}_{2} \times \mathbf{e}_{3}$ is the Jacobian. $\Phi(\rho)$ and $\psi(\rho)$ are, respectively, $1 / 2 \pi$ times the toroidal magnetic flux inside, and the poloidal flux outside, flux surface $\rho$, and $J(\rho)$ and $F(\rho)$ are, respectively, $\mu_{0} / 2 \pi$ times the toroidal current inside, and the poloidal current outside, flux surface $\rho$. Using Eq.(2) in Ampere's law, one obtains the contravariant representation of the current,

$$
\mu_{0} \mathbf{J}=\nabla \times \mathbf{B}=-\left(F^{\prime}+\partial_{\zeta} \nu\right) \nabla \zeta \times \nabla \rho+\left(J^{\prime}+\partial_{\theta} \nu\right) \nabla \rho \times \nabla \theta .
$$

Eqs.(1) and (3) automatically satisfy $\nabla \cdot \mathbf{B}=0$ and the steady-state condition $\nabla \cdot \mathbf{J}=0$, as well as the equilibrium conditions $B^{\rho} \equiv \nabla \rho \cdot \mathbf{B}=0$ and $J^{\rho} \equiv \nabla \rho \cdot$ $\mathbf{J}=0$ arising from dotting $\mathbf{B}$ and $\mathbf{J}$ into the force-balance equation $p^{\prime} \nabla \rho=\mathbf{J} \times \mathbf{B}$. The final equilibrium condition is the radial component of this,

$$
|\nabla \rho|^{2} p^{\prime}=\nabla \rho \cdot \mathbf{J} \times \mathbf{B}
$$


The standard (2D) GS equation uses neither the co- nor the contra-variant representations of $\mathbf{B}$ and $\mathbf{J}$ in Eq.(4), but the 'mixed' representation

$$
\mathbf{B}=\nabla \psi \times \mathbf{b}+\mathbf{b} F, \mu_{0} \mathbf{J}=-\mathbf{b} \Delta^{*} \psi+\nabla F \times \mathbf{b}
$$

where $\mathbf{b} \equiv \nabla \zeta_{g}=\hat{\zeta} / R$, and $\mathbf{b}^{2} \Delta^{*} \psi \equiv \nabla \cdot\left(\mathbf{b}^{2} \nabla \psi\right)$. Using Eqs.(5) in (4), one obtains

$$
\mathbf{b}^{2} \Delta^{*} \psi=-\mu_{0} p^{\prime} / \psi^{\prime}-\mathbf{b}^{2} F F^{\prime} / \psi^{\prime}
$$

Axisymmetry has been used in obtaining the simple forms for $\mathbf{J}$ and Eq.(6). Specializing $\rho$ to $\psi$ here so that $\psi^{\prime}=1$, and noting that $\mathbf{b}^{2}=R^{-2}$ yields the GS equation.

In the fully 3D problem, Degtyarev, et al. ${ }^{7}$ have shown that a mixed representation may again be given, making use of two special flux coordinate systems, the 'natural' and 'conatural' systems $\left(\rho, \theta_{n}, \zeta_{n}\right)$ and $\left(\rho, \theta_{c}, \zeta_{c}\right)$, respectively, which become the same system in the $2 \mathrm{D}$ case. Demanding $\nabla \rho \cdot \nabla \times \mathbf{B}=0$ from representation (1) for $\mathbf{B}$, and further that this condition hold independent of the rotational transform $\iota \equiv-\psi^{\prime} / \Phi^{\prime}$ results in equations determining the angles for the natural coordinate system:

$$
\nabla \cdot\left[\nabla \rho \times\left(\nabla \theta_{n} \times \nabla \rho\right)\right]=0, \nabla \cdot\left[\nabla \rho \times\left(\nabla \zeta_{n} \times \nabla \rho\right)\right]=0,
$$

and demanding $\nabla \cdot \mathbf{B}=0$ from representation (2) for the conatural system, and further that this hold independent of the ratio $F / J$ results in similar conditions determining the angles for the conatural system:

$$
\nabla \cdot\left[\nabla \rho \times\left(\nabla \theta_{c} \times \nabla \rho\right) /|\nabla \rho|^{2}\right]=0, \nabla \cdot\left[\nabla \rho \times\left(\nabla \zeta_{c} \times \nabla \rho\right) /|\nabla \rho|^{2}\right]=0
$$

Each of Eqs.(7) and (8) have no radial derivatives, and so are 2D p.d.e.s over a flux surface. Starting only with $\rho(\mathbf{x})$, one uses Eqs.(7) and (8) to obtain the full natural 
and conatural coordinate sets. Given these, the generalized mixed representation for $\mathbf{B}$ is shown in Ref. 7 to be

$$
\mathbf{B}=\nabla \psi \times \mathbf{b}_{c}+\mathbf{b}_{n} F
$$

where $\mathbf{b}_{c} \equiv \mathbf{e}_{c 3} /\left(\mathbf{e}_{c 3} \cdot \mathbf{e}_{n 3}\right)$ and $\mathbf{b}_{n} \equiv \mathbf{e}_{n 3} /\left(\mathbf{e}_{c 3} \cdot \mathbf{e}_{n 3}\right)$. The current is then given by

$$
\mu_{0} \mathbf{J}=\nabla \times\left(\nabla \psi \times \mathbf{b}_{c}\right)+\nabla F \times \mathbf{b}_{n}+F \nabla \times \mathbf{b}_{n}
$$

where the final term vanishes in the 2D case. Using Eqs.(9) and (10) in (4) yields the 3D-GS equation,

$$
\begin{aligned}
\mathbf{b}_{c}^{2} \Delta_{c}^{*} \psi= & -\mu_{0} p^{\prime} / \psi^{\prime}-\mathbf{b}_{n}^{2} F F^{\prime} / \psi^{\prime}+F \mathbf{b}_{c} \cdot \nabla \times \mathbf{b}_{n}-F^{\prime} \mathbf{b}_{c} \cdot\left(\mathbf{b}_{n} \times \nabla \rho\right) \\
& -\left(F / \psi^{\prime}|\nabla \rho|^{2}\right)\left(\mathbf{b}_{n} \times \nabla \rho\right) \cdot \nabla \times\left(\mathbf{b}_{c} \times \nabla \psi\right)+\left(F^{2} / \psi^{\prime}|\nabla \rho|^{2}\right)\left(\mathbf{b}_{n} \times \nabla \rho\right) \cdot\left(\nabla \times \mathbf{b}_{n}\right),
\end{aligned}
$$

where the operator $\Delta_{c}^{*}$ generalizes $\Delta^{*}$ in the GS equation:

$\mathbf{b}_{c}^{2} \Delta_{c}^{*} \psi \equiv-\mathbf{b}_{c} \cdot \nabla \times\left(\nabla \psi \times \mathbf{b}_{c}\right)=\nabla \cdot\left(\mathbf{b}_{c}^{2} \nabla \psi\right)-\nabla \psi \cdot \mathbf{b}_{c} \times\left(\nabla \times \mathbf{b}_{c}\right)$. Of the 6 terms on the right side of Eq.(11), all but the first 2 vanish in the axisymmetric case (6), as does the second term in last form given for $\mathbf{b}_{c}^{2} \Delta_{c}^{*} \psi$.

We now show that Eq.(11) has a form amenable to the analysis to which CT subjected (6) in Ref. 1. Using $\rho$ as the radial variable, we write each of the terms in $\psi^{\prime} \times$ Eq.(11) as the product of some combination of the physics-related profile functions $\psi(\rho), p(\psi)$ and $F(\rho)$ and their derivatives, times a geometric coefficient $\left(A(\mathbf{x}), C(\mathbf{x}), D_{i=0-4}(\mathbf{x})\right)$ which varies over a flux surface:

$$
\psi^{\prime} \psi^{\prime \prime} A+\psi^{\prime 2} C=-p^{\prime} D_{0}-F F^{\prime} D_{1}-F \psi^{\prime} D_{2}-\left(F \psi^{\prime}\right)^{\prime} D_{3}-F^{2} D_{4},
$$

where $A \equiv \mathbf{b}_{c}^{2}|\nabla \rho|^{2}, C \equiv \mathbf{b}_{c}^{2} \Delta_{c}^{*} \rho, D_{0} \equiv \mu_{0}, D_{1} \equiv \mathbf{b}_{n}^{2}, D_{2} \equiv\left[-\mathbf{b}_{c} \cdot \nabla \times \mathbf{b}_{n}+\right.$ $\left.\left(\mathbf{b}_{n} \times \nabla \rho\right) \cdot \nabla \times\left(\mathbf{b}_{c} \times \nabla \rho\right) /|\nabla \rho|^{2}\right], D_{3} \equiv \mathbf{b}_{c} \cdot\left(\mathbf{b}_{n} \times \nabla \rho\right)$, and $D_{4} \equiv-\left(\mathbf{b}_{n} \times\right.$ $\nabla \rho) \cdot\left(\nabla \times \mathbf{b}_{n}\right) /|\nabla \rho|^{2}$. Only the coefficients $A, C, D_{0}$ and $D_{1}$ are nonvanishing in the $2 \mathrm{D}$ case. 
As in Ref. 1, we note that Eq.(12) has an immense amount of redundancy: an infinite number of coupled o.d.e.s in $\rho$ may be generated from it by taking different flux surface averages. For example, for any "test functions" $h_{i}(\mathbf{x}),(i=0-4)$ with vanishing flux surface average, $\left\langle h_{i}\right\rangle=0$, taking $\left\langle(12) \times h_{i} / D_{i}\right\rangle$ yields a 1D equation of the form of Eq.(12), but with the term in $D_{i}$ annihilated. Generating such an o.d.e. for each of the $5 D_{i}$, one obtains a set of 5 linear equations in the 7 'unknowns' $\left\{\psi^{\prime} \psi^{\prime \prime}, \psi^{\prime 2} ; p^{\prime}, F F^{\prime}, F \psi^{\prime},\left(F \psi^{\prime}\right)^{\prime}, F^{2}\right\}$. Thus, by taking linear combinations, one can eliminate the last 5 of these, and obtain an equation of the same form as found in Ref. 1,

$$
\psi^{\prime} \psi^{\prime \prime} A_{T}+\psi^{\prime 2} C_{T}=0
$$

which is easily solved for $\psi(\rho)$. Calling the coefficient of the $i^{\text {th }}$ equation $\alpha_{i},(i=$ $0-4)$, Eq.(13) is thus obtained by the flux surface average $\left\langle(12) \times h_{T}\right\rangle$, with $h_{T}(\mathbf{x}) \equiv \sum_{i=0}^{4} \alpha_{i} h_{i}(\mathbf{x}) / D_{i}(\mathbf{x})$. Given $\psi$, one may obtain any of the other flux function unknowns, and thus $p^{\prime}$ and $F$, through other combinations of the 5 o.d.e.s. A slightly different approach is to make contact with the starting point of CT for this part of the analysis, the GS equation written with $\rho$ as the radial variable. As noted above, this is just Eq.(12) with vanishing $D_{2}, D_{3}$, and $D_{4}$. Using only the last $3(i=2,3,4)$ of the 5 averaged equations above, one can straightforwardly eliminate the unknowns $F \psi^{\prime},\left(F \psi^{\prime}\right)^{\prime}$, and $F^{2}$ from Eq.(12), obtaining

$$
\psi^{\prime} \psi^{\prime \prime} \tilde{\tilde{A}}+\psi^{\prime 2} \tilde{\tilde{C}}=-p^{\prime} \tilde{\tilde{D}}_{0}-F F^{\prime} \tilde{\tilde{D}}_{1}
$$

where all 4 coefficients $\tilde{\tilde{X}}$ here are given by $\tilde{\tilde{X}} \equiv \tilde{X}-\left\langle\tilde{X} h_{1} / \tilde{D}_{1}\right\rangle \tilde{D}_{4} /\left\langle\tilde{D}_{4} h_{1} / \tilde{D}_{1}\right\rangle$, and $\tilde{X} \equiv X-\left\langle X h_{2} / D_{2}\right\rangle D_{3} /\left\langle D_{3} h_{2} / D_{2}\right\rangle-\left\langle X h_{3} / D_{3}\right\rangle D_{2} /\left\langle D_{2} h_{3} / D_{3}\right\rangle$. Eq.(14) is of the same form as the GS equation, but with the replacements $\mathbf{b}^{2}|\nabla \rho|^{2} \rightarrow \tilde{\tilde{A}}$, $\mathbf{b}^{2} \Delta^{*} \rho \rightarrow \tilde{\tilde{C}}, \mu_{0} \rightarrow \tilde{\tilde{D}}_{0}$, and $\mathbf{b}^{2} \rightarrow \tilde{\tilde{D}}_{1}$. Thus, the same expressions given in Ref. 1 for $\psi, p^{\prime}$ and $F F^{\prime}$ apply here as well, with these replacements. 
Summarizing, we have shown that the $2 \mathrm{D}$ result of Ref. 1, that knowing only the shape $\rho(\mathbf{x})$ of the flux surfaces in a toroidal MHD equilibrium is sufficient to determine the full equilibrium, can be extended to 3D equilibria, such as those of stellarators. This is achieved by building on the results of Ref. 7, which showed that a 3D analog for the GS equation exists, and by demonstrating that this 3D-GS equation retains the needed properties for the CT method to be applied. As noted in Ref. 1, the equilibrium equation has a great deal of redundancy, reflected in the great flexibility in the choice of the test functions $h_{i}(\mathbf{x})$. These may be chosen to be appreciable everywhere over each flux surface, or highly localized, depending, for example, on what type of data one has available to determine $\rho(\mathbf{x})$. The fact that $\rho(\mathbf{x})$ describes a 3D equilibrium assures that any choice will yield the same result. However, if the precision with which this information is known is limited, as will be the case if $\rho(\mathbf{x})$ is measured experimentally, a corresponding spread in the results for $\psi, p^{\prime}$ and $F F^{\prime}$ will arise for different choices of $h_{i}$. Study of this, and the practicality of the $\mathrm{CT}$ approach to profile determination in stellarators, are left to future work.

\section{Acknowledgment}

This work supported by U.S.Department of Energy Contract No.DE-AC02-76CHO3073. 


\section{References}

${ }^{1}$ J.P. Christiansen, J.B. Taylor, Nucl.Fusion 22, 111 (1982).

${ }^{2} \mathrm{H}$. Grad, H. Rubin, Proceedings of the 2nd United Nations International Conference on the Peaceful Uses of Atomic Energy 31190 (1958).

${ }^{3}$ V.D. Shafranov, Sov. Phys. JETP 6, 545 (1958).

${ }^{4}$ J.P. Christiansen, J.D. Callen, J.J. Ellis, R.S. Granetz, Nucl. Fusion 29703 (1989).

${ }^{5}$ R.S. Granetz, M.C. Borrás, Fusion and Engr. Design 34-35 153 (1997).

${ }^{6}$ K. Tritz, R. Fonck, T. Thorson, Rev. Scientific Instruments 70595 (1999).

${ }^{7}$ L.M. Degtyarev, V.V. Drozdov, M.I. Mikhailov, V.D. Pustovitov, V.D. Shafranov, Sov. J. Plasma Phys. 1122 (1985). 


\section{External Distribution}

Plasma Research Laboratory, Australian National University, Australia

Professor I.R. J ones, Flinders University, Australia

Professor J oão Canalle, Instituto de Fisica DEQ/IF - UERJ , Brazil

Mr. Gerson O. Ludwig, Instituto Nacional de Pesquisas, Brazil

Dr. P.H. Sakanaka, Instituto Fisica, Brazil

The Librarian, Culham Laboratory, England

Library, R61, Rutherford Appleton Laboratory, England

Mrs. S.A. Hutchinson, JET Library, England

Professor M.N. Bussac, Ecole Polytechnique, France

Librarian, Max-Planck-Institut für Plasmaphysik, Germany

J olan Moldvai, Reports Library, MTA KFKI-ATKI, Hungary

Dr. P. Kaw, Institute for Plasma Research, India

Ms. P.J . Pathak, Librarian, Insitute for Plasma Research, India

Ms. Clelia De Palo, Associazione EURATOM-ENEA, I taly

Dr. G. Grosso, Instituto di Fisica del Plasma, Italy

Librarian, Naka Fusion Research Establishment, J AERI, J apan

Library, Plasma Physics Laboratory, Kyoto University, J apan

Research Information Center, National Institute for Fusion Science, J apan

Dr. O. Mitarai, Kyushu Tokai University, J apan

Library, Academia Sinica, Institute of Plasma Physics, People's Republic of China

Shih-Tung Tsai, Institute of Physics, Chinese Academy of Sciences, People's Republic of China

Dr. S. Mirnov, TRINITI, Troitsk, Russian Federation, Russia

Dr. V.S. Strelkov, Kurchatov Institute, Russian Federation, Russia

Professor Peter Lukac, Katedra Fyziky Plazmy MFF UK, Mlynska dolina F-2, Komenskeho Univerzita, SK-842 15 Bratislava, Slovakia

Dr. G.S. Lee, Korea Basic Science Institute, South Korea

Mr. Dennis Bruggink, Fusion Library, University of Wisconsin, USA

Institute for Plasma Research, University of Maryland, USA

Librarian, Fusion Energy Division, Oak Ridge National Laboratory, USA

Librarian, Institute of Fusion Studies, University of Texas, USA

Librarian, Magnetic Fusion Program, Lawrence Livermore National Laboratory, USA

Library, General Atomics, USA

Plasma Physics Group, Fusion Energy Research Program, University of California at San Diego, USA

Plasma Physics Library, Columbia University, USA

Alkesh Punjabi, Center for Fusion Research and Training, Hampton University, USA

Dr. W.M. Stacey, Fusion Research Center, Georgia Institute of Technology, USA

Dr. J ohn Willis, U.S. Department of Energy, Office of Fusion Energy Sciences, USA

Mr. Paul H. Wright, Indianapolis, Indiana, USA 
The Princeton Plasma Physics Laboratory is operated by Princeton University under contract with the U.S. Department of Energy.

\author{
Information Services \\ Princeton Plasma Physics Laboratory \\ P.O. Box 451 \\ Princeton, NJ 08543
}

Phone: 609-243-2750

Fax: 609-243-2751

e-mail: pppl_info@pppl.gov

Internet Address: http://www.pppl.gov 